\title{
Efecto de la inclusión de Azolla en la dieta de codornices japonesas sobre el consumo voluntario, digestibilidad aparente y producción de huevos
}

\author{
EFFECT OF AZOLLA INCLUSION IN THE DIET OF JAPANESE QUAILS ON VOLUNTARY INTAKE, \\ APPARENT DIGESTIBILITY AND EGG PRODUCTION
Juan Buenaño-Buenaño ${ }^{1}$, Patricio Nuñez-Torres ${ }^{1}$, Marcos Barros-Rodríguez ${ }^{1,2}$, Hernán Zurita-Vásquez ${ }^{1}$ \\ Marco Rosero-Peñaherrera ${ }^{1}$, Efraín Lozada-Salcedo ${ }^{1}$, Carlos Guishca-Cunuhay ${ }^{1}$,
}

\section{Resumen}

\begin{abstract}
El objetivo de esta investigación fue evaluar el efecto de la inclusión de Azolla en la dieta de codornices japonesas sobre el consumo voluntario, digestibilidad aparente de los nutrientes y producción de huevos. Se utilizaron 240 codornices de 16 semanas de edad iniciando el primer tercio de postura. Las aves se alojaron en 24 jaulas elevadas (10 aves por jaula). Se utilizó un diseño completamente al azar con cuatro tratamientos: T1: 0\%, T2: 5\%, T3: 10\% y T4: 15\% de inclusión de Azolla en la dieta por 60 días. El consumo voluntario de nutrientes fue mayor en los tratamientos con Azolla $(\mathrm{p}<0.05)$. El menor rendimiento de huevos $\mathrm{g} / \mathrm{ave} / \mathrm{día}^{1}$ fue en $\mathrm{T} 4(\mathrm{p}<0.0001)$ y la mejor conversión alimenticia fue en T1 y T2 $(\mathrm{p}<0.0001)$. La digestibilidad aparente de la materia seca y materia orgánica no mostró diferencia significativa entre tratamientos. La mayor digestibilidad de proteína cruda fue en T1 $(46.1 \%)$ y T2 $(44.5 \%)(\mathrm{p}<0.0001)$, y para fibra detergente neutra fue en T2 $(63.6 \%)(\mathrm{p}=0.034)$. La incorporación de Azolla en un 5\% en la dieta de codornices produce un efecto similar en la producción de huevos y conversión alimenticia que la dieta control, pudiendo utilizarse como un producto no convencional de bajo costo.
\end{abstract}

Palabras clave: Azolla; codorniz; digestibilidad; consumo voluntario

\section{Abstract}

The aim of this study was to evaluate the effect of the inclusion of Azolla on the diet of Japanese quails on voluntary intake, apparent nutrient digestibility and egg production. A total of 240 16-week old quail on the first third of egg laying were used. The birds were

${ }^{1}$ Facultad de Ciencias Agropecuarias, Universidad Técnica de Ambato, Cevallos, Ecuador

${ }^{2}$ E-mail:ma_barrosr@yahoo.es

Recibido: 26 de marzo de 2017

Aceptado para publicación: 8 de noviembre de 2017 
housed in 24 raised cages (10 per cage). A completely randomized design was used with four treatments: T1: 0\%, T2: 5\%, T3: 10\% and T4: 15\% inclusion of Azolla in the diet. Voluntary nutrient intake was higher in Azolla treatments $(\mathrm{p}<0.05)$. The lowest egg yield $\mathrm{g} /$ bird/day ${ }^{1}$ was in T4 $(\mathrm{p}<0.0001)$ and the best feed conversion was in T1 and T2 $(\mathrm{p}<0.0001)$. The apparent digestibility of dry matter and organic matter showed no significant difference between treatments. The highest crude protein digestibility was in T1 $(46.1 \%)$ and T2 $(44.5 \%)(\mathrm{p}<0.0001)$ and the highest for digestibility of neutral detergent fibre was in T2 $(63.6 \%)(\mathrm{p}=0.034)$. The incorporation of Azolla by $5 \%$ in the quail diet produces a similar effect in egg production and feed conversion than the control diet, which could be used as a non-conventional low-cost product.

Key words: Azolla; quail; digestibility; voluntary intake

\section{INTRODUCCIÓN}

La codorniz japonesa (Coturnix coturnix japonica) viene generando gran interés en los avicultores dedicados a la produccion de huevos en el Ecuador, dado que es un ave que requiere de poca inversión económica para su crianza, en comparación con otras aves de granja (Özsoy y Aktan, 2011). La codorniz es la especie de menor contextura que se explota en el sector avícola comercial (Panda y Singh, 1990). La razón de su éxito se explica por el alto nivel de producción y la calidad nutricional de los huevos (Iqbal et al., 2015) que ha dado lugar a su aceptación en el mercado de consumo.

Las hembras en crianza comercial inician su ciclo de postura, generalmente, a las seis semanas de edad y pueden llegar a mantener un promedio de 250-300 huevos por campaña (Dahouda et al., 2013). La calidad del huevo juega un papel importante en la crianza de la codorniz, puesto que depende de ello la demanda del producto (Kocevski et al., 2011), de allí que el éxito financiero está en el número total de huevos (Monira et al., 2003). Sin embargo, el productor se enfrenta a una serie de dificultades que limitan una producción eficiente, al no contar con alimentos balanceados definidos para su alimentación. Como alternativa para este problema se plantea la formulación de concentrados a base de productos no convencionales que mejoren la ingesta de alimento, su digestibilidad y la producción de huevos.

En este sentido, la incorporación de Azolla en la dieta de codornices puede jugar un papel muy importante, debido a que posee entre 17 a $18 \%$ de proteína, bajos niveles de fibra (34\% de fibra detergente neutro - FDN) y alrededor de $90 \%$ de digestibilidad de la materia seca (MS) (Becerra y Preston, 1990; Vásquez, 2012). La Azolla ha sido utilizada en la alimentación animal, tanto como forraje fresco para rumiantes como en harina para la elaboración de alimentos balanceados de monogástricos, obteniendo buenos rendimientos productivos al sustituir de forma parcial o total la soya y maíz, respectivamente (Ramos, 2012; Vásquez, 2012).

La utilización de harinas a base de forrajes puede ser promisorio en la producción de codornices ya que, según Çabuk et al. (2014), se pueden lograr incrementos significativos de la producción de huevos al usar inclusiones de $10-20 \%$ de subproductos de lenteja (Lens culinaris) en la alimentación de codornices. Así mismo, indican que la incorporación de niveles que excedan los límites recomendados tiende a disminuir el consumo y la conversión del alimento en carne y huevo. Esto se debe a la presencia de niveles excedentes de carbohidratos estructurales en la dieta, que provoca un pasaje lento del alimento en el tracto gastrointestinal aunado a un mayor gasto energético (Savory y Gentle, 
1976; Vieira Filho et al., 2016). Con base en estos antecedentes, el objetivo de esta investigación fue evaluar el efecto de la inclusión de Azolla en la alimentación de codornices japonesas sobre el consumo voluntario, la digestibilidad aparente de los nutrientes y la producción de huevos.

\section{Materiales y Métodos}

\section{Ubicación, Animales y Alojamiento}

La presente investigación se realizó en la Facultad de Ciencias Agropecuarias de la Universidad Técnica de Ambato (UTA), Ecuador. La zona se encuentra a una altitud de $2865 \mathrm{msnm}$.

Se utilizaron 240 codornices (Coturnix coturnix japonica) de aproximadamente 16 semanas de edad que se encontraban en el primer tercio de postura. Las codornices provenían de una granja donde su alimentación estaba basada en balanceado comercial. Las aves estaban alojadas en 24 jaulas elevadas (10 aves por jaula), provistas de comederos y bebederos con agua ad libitum. Las codornices tuvieron un periodo de adaptación de 30 días, donde el alimento concentrado se fue mezclando con las dietas experimentales, hasta solo proporcionar las dietas a base de niveles crecientes de Azolla. Finalizado el periodo de adaptación se inició con los periodos de muestreos cada 15 días durante 60 días.

Las codornices se distribuyeron de manera aleatoria en los cuatro tratamientos.

\section{Azolla y Tratamientos}

La Azolla fue cultivada en los estanques de la Facultad de Ciencias Agropecuarias, UTA. Se recolectaron $1500 \mathrm{~kg}$ de materia fresca a las cuatro semanas de la siembra. La Azolla recolectada fue deshidratada al sol bajo cubierta de invernadero. Una vez seca, se molió en un molino de martillo a un tamaño de partícula de $1 \mathrm{~mm}$ y se incorporó en la dieta de las codornices según los niveles establecidos para cada tratamiento experimental (dietas) (Cuadro 1).

Adicionalmente, se tomó una muestra de $1 \mathrm{~kg}$ de materia fresca para estimar el contenido de materia seca (MS) en una estufa a $60{ }^{\circ} \mathrm{C}$ hasta peso constante, para luego realizar el análisis bromatológico.

El alimento fue suministrado dos veces por día (10:00 y 16:00 h) durante todo el periodo experimental. Se tomaron $200 \mathrm{~g}$ de cada dieta y se secaron en una estufa a $60^{\circ} \mathrm{C}$ para determinar la MS de las dietas y la composición química.

\section{Variables de Respuesta}

Consumo voluntario de nutrientes. Se determinó por el método directo (nutriente ofrecido - nutriente rechazado) cada 24 horas durante tres días consecutivos y cada 15 días $(0,15,30,45$ y 60 del periodo experimental).

Rendimiento de huevos (g/día) y conversión alimenticia. Se registró el número de huevos por jaula cada 24 horas por tres días consecutivos y cada 15 días hasta finalizar el periodo experimental. Los huevos y el alimento fueron pesados en una balanza de precisión PCE-BT 2000 (0.01 g de precisión). La conversión alimenticia se determinó mediante la división entre el alimento consumido y el rendimiento de huevos.

Digestibilidad aparente de los nutrientes. Se realizó mediante el método directo de recolección total de heces. Se determinó mediante la fórmula: nutriente ingerido - nutriente excretado cada 24 horas. Este procedimiento se realizó durante seis días al final del periodo experimental (día 61 a 67). Para esto, se recolectó diariamente una muestra del alimento ofrecido y de las heces por jaula, se secaron estufa a $60{ }^{\circ} \mathrm{C}$ para determinar la MS y el contenido de nutrientes, tanto del ingerido (alimento) como del rechazado (en las heces). 
Cuadro 1. Niveles de inclusión de Azolla y composición química de las dietas experimentales

\begin{tabular}{|c|c|c|c|c|}
\hline \multirow{2}{*}{$\begin{array}{l}\text { Ingredientes } \\
(\mathrm{g} / \mathrm{kg} \mathrm{MS})\end{array}$} & \multicolumn{4}{|c|}{ Tratamientos } \\
\hline & $\mathrm{T} 1$ & $\mathrm{~T} 2$ & $\mathrm{~T} 3$ & $\mathrm{~T} 4$ \\
\hline Maíz & 446.0 & 442.0 & 400.0 & 365.0 \\
\hline Aceite de palma & 36.0 & 37.0 & 42.0 & 36.0 \\
\hline Afrecho & 60.0 & 15.0 & 4.0 & 10.0 \\
\hline Pasta de soya & 340.0 & 340.0 & 335.0 & 320.0 \\
\hline Harina de pescado & 25.0 & 23.0 & 26.0 & 26.0 \\
\hline Azolla & 0 & 50.0 & 100.0 & 150.0 \\
\hline Melaza & 10.0 & 10.0 & 10.0 & 10.0 \\
\hline Lisina & 0.50 & 0.50 & 0.50 & 0.50 \\
\hline Metionina & 1.20 & 1.20 & 1.20 & 1.20 \\
\hline Treonina & 0.80 & 0.80 & 0.80 & 0.80 \\
\hline Fosfato monodicálcico & 12.0 & 12.0 & 12.0 & 12.0 \\
\hline Carbonato de calcio & 63.0 & 63.0 & 63.0 & 63.0 \\
\hline Sal común & 3.0 & 3.0 & 3.0 & 3.0 \\
\hline Vitaminas & 2.0 & 2.0 & 2.0 & 2.0 \\
\hline Total & 1000 & 1000 & 1000 & 1000 \\
\hline \multicolumn{5}{|l|}{ Composición química (\%) } \\
\hline Proteína cruda (PC) & 22.9 & 22.2 & 22.4 & 23.7 \\
\hline Materia seca (MS) & 89.1 & 89.4 & 89.1 & 89.2 \\
\hline Materia orgánica (MO) & 92.0 & 90.4 & 88.2 & 90.0 \\
\hline Fibra detergente neutro (FDN) & 25.1 & 24.7 & 24.0 & 25.2 \\
\hline Fibra detergente ácido (FDA) & 22.1 & 19.5 & 21.0 & 22.6 \\
\hline Cenizas & 8.8 & 9.5 & 11.7 & 9.9 \\
\hline
\end{tabular}

\section{Análisis Químicos}

Se determinó la MS (\#7.007), PC (\#2.057) y ceniza (\#7.009) acorde a la metodología descrita por AOAC (1990). La fibra detergente ácida (FDA) y fibra detergente neutra (FDN) se determinó de acuerdo a los métodos 12 y 13, respectivamente, del analizador de fibra ANKOM ${ }^{2000}$ (Ankom Technology, Fairport, EEUU). El contenido de proteína cruda $(\mathrm{PC})$ se realizó por análisis elemental (N) utilizando un LECO CHN 628 (LECO Corporation).

\section{Diseño Experimental y Análisis Estadís- tico}

Se utilizó un diseño completamente al azar con cuatro tratamientos (dietas) y seis repeticiones (jaulas con 10 aves). Todas las 
Cuadro 2.Consumo voluntario de nutrientes ( $\left.\mathrm{g} / \mathrm{ave}_{\mathrm{d}} \mathrm{dia}^{-1}\right)$, rendimiento de huevos (g/ave/día $\left.{ }^{-1}\right)$ y conversión alimenticia en codornices alimentadas por 60 días con niveles crecientes de Azolla

\begin{tabular}{lcccccccc}
\hline \multirow{2}{*}{$\begin{array}{l}\text { Consumo de } \\
\text { nutrientes }\end{array}$} & \multicolumn{5}{c}{ Tratamientos } & \multirow{2}{*}{ EEM } & p & \multicolumn{2}{c}{ Contrastes } \\
\cline { 2 - 4 } \cline { 7 - 9 } & $\mathrm{T} 1$ & $\mathrm{~T} 2$ & $\mathrm{~T} 3$ & $\mathrm{~T} 4$ & & & $\mathrm{~L}$ & $\mathrm{C}$ \\
\hline $\mathrm{MS}$ & $41.0^{\mathrm{B}}$ & $44.6^{\mathrm{AB}}$ & $47.4^{\mathrm{A}}$ & $45.2^{\mathrm{A}}$ & 0.934 & 0.0010 & 0.6619 & 0.0422 \\
$\mathrm{MO}$ & $37.7^{\mathrm{B}}$ & $40.3^{\mathrm{AB}}$ & $41.8^{\mathrm{A}}$ & $40.7^{\mathrm{AB}}$ & 0.835 & 0.0172 & 0.7740 & 0.2248 \\
$\mathrm{PC}$ & $9.4^{\mathrm{B}}$ & $9.9^{\mathrm{AB}}$ & $10.6^{\mathrm{A}}$ & $10.7^{\mathrm{A}}$ & 0.211 & 0.0007 & 0.0137 & 0.2488 \\
$\mathrm{FDN}$ & $10.3^{\mathrm{B}}$ & $11.0^{\mathrm{AB}}$ & $11.3^{\mathrm{A}}$ & $11.4^{\mathrm{A}}$ & 0.229 & 0.0099 & 0.2765 & 0.5757 \\
FDA & $9.0^{\mathrm{B}}$ & $8.7^{\mathrm{B}}$ & $9.9^{\mathrm{A}}$ & $10.2^{\mathrm{A}}$ & 0.199 & 0.0001 & 0.0001 & 0.0532 \\
$\begin{array}{l}\text { Rendimiento } \\
\text { de huevos }\end{array}$ & $11.8^{\mathrm{A}}$ & $11.4^{\mathrm{A}}$ & $10.7^{\mathrm{A}}$ & $9.1^{\mathrm{B}}$ & 0.333 & 0.0001 & 0.0001 & 0.3099 \\
Conversión & & & & & & & & \\
alimenticia & $3.4^{\mathrm{C}}$ & $3.8^{\mathrm{CB}}$ & $4.4^{\mathrm{AB}}$ & $5.0^{\mathrm{A}}$ & 0.163 & 0.0001 & 0.0002 & 0.9321 \\
\hline
\end{tabular}

$A B C$ Medias con letras diferentes dentro de filas difieren significativamente $(p<0.05)$

$\mathrm{EEM}=$ Error estándar de la media; $\mathrm{MS}$ = Materia seca; $\mathrm{MO}$ = Materia orgánica; $\mathrm{PC}$ = Proteína cruda; FDN = Fibra detergente neutra; FDA = Fibra detergente ácida

$\mathrm{T} 1=0 \%, \mathrm{~T} 2=5 \%, \mathrm{~T} 3=10 \%, \mathrm{~T} 4=15 \%$ de inclusión de Azolla en la dieta

Cuadro 3. Digestibilidad aparente de los nutrientes en dietas con niveles crecientes de Azolla utilizadas en la alimentación de codornices

\begin{tabular}{|c|c|c|c|c|c|c|c|c|}
\hline \multirow{2}{*}{$\begin{array}{l}\text { Digestibilidad } \\
\text { aparente }(\%)\end{array}$} & \multicolumn{4}{|c|}{ Tratamientos } & \multirow{2}{*}{ EEM } & \multirow{2}{*}{$\mathrm{p}$} & \multicolumn{2}{|c|}{ Contrastes } \\
\hline & $\mathrm{T} 1$ & $\mathrm{~T} 2$ & T3 & $\mathrm{T} 4$ & & & $\mathrm{~L}$ & $\mathrm{C}$ \\
\hline MS & $65.4^{\mathrm{A}}$ & $70.2^{\mathrm{A}}$ & $69.6^{\mathrm{A}}$ & $68.5^{\mathrm{A}}$ & 1.26 & 0.0593 & 0.3402 & 0.8589 \\
\hline MO & $69.6^{\mathrm{A}}$ & $73.4^{\mathrm{A}}$ & $72.7^{\mathrm{A}}$ & $72.5^{\mathrm{A}}$ & 1.10 & 0.1086 & 0.5408 & 0.8450 \\
\hline $\mathrm{PC}$ & $46.1^{\mathrm{A}}$ & $44.5^{\mathrm{A}}$ & $33.8^{\mathrm{B}}$ & $37.4^{\mathrm{B}}$ & 2.31 & 0.0001 & 0.0429 & 0.0195 \\
\hline FDN & $56.1^{\mathrm{B}}$ & $63.6^{\mathrm{A}}$ & $54.3^{\mathrm{B}}$ & $55.4^{\mathrm{B}}$ & 1.68 & 0.0034 & 0.0025 & 0.0206 \\
\hline FDA & $52.4^{\mathrm{AB}}$ & $55.0^{\mathrm{A}}$ & $45.9^{\mathrm{B}}$ & $49.9^{\mathrm{AB}}$ & 1.91 & 0.0205 & 0.0725 & 0.0113 \\
\hline
\end{tabular}

$A B C$ Medias con letras diferentes dentro de filas difieren significativamente $(p<0.05)$

$\mathrm{EEM}=$ Error estándar de la media; $\mathrm{MS}$ = Materia seca; $\mathrm{MO}$ = Materia orgánica; $\mathrm{PC}$ = Proteína cruda;

FDN = Fibra detergente neutra; FDA = Fibra detergente ácida

$\mathrm{T} 1=0 \%, \mathrm{~T} 2=5 \%, \mathrm{~T} 3=10 \%, \mathrm{~T} 4=15 \%$ de inclusión de Azolla en la dieta 
variables fueron analizadas de acuerdo al diseño empleado usando el PROC GLM (SAS, 2002). La comparación de medias se lo realizó mediante la prueba de Tukey. Adicionalmente, se realizó un análisis de polinomios ortogonales para evaluar los efectos lineales o cuadráticos de la respuesta a los tratamientos (SAS, 2002).

\section{Resultados}

El consumo de MS, materia orgánica (MO), PC y FDN fue mayor ( $\mathrm{p}=0.0010$, $\mathrm{p}=0.0172, \mathrm{p}=0,0007, \mathrm{p}=0,0099$, respectivamente) para los tratamientos $\mathrm{T} 2$, $\mathrm{T} 3$ y T4 en comparación a $\mathrm{T} 1$, obteniendo una respuesta cuadrática hacia el efecto de la Azolla en el consumo de la MS ( $\mathrm{p}=0.0422)$ y lineal en la ingestión de $\mathrm{PC}(\mathrm{p}=0.0137)$.

El consumo de FDA presenta una respuesta lineal $(\mathrm{p}<0.0001)$ hacia los tratamientos, obteniendo el mayor consumo voluntario en las aves alimentadas con T3 y T4 $(p=0.0001)$. El menor rendimiento de huevos $\mathrm{g} /$ ave/día ${ }^{1}$ y conversión alimenticia se observó en T4 ( $p<0.0001)$, obteniendo una respuesta lineal $(\mathrm{p}<0.0001)$ sobre el efecto de los tratamientos; es decir, a mayor nivel de Azolla en la dieta se disminuye el rendimiento de huevos y la conversión alimenticia (Cuadro 2).

La digestibilidad aparente de la MS y MO no mostró diferencia entre los tratamientos $(\mathrm{p}=0.0593$ y $\mathrm{p}=0.1086$, respectivamente). Con respecto a la $\mathrm{PC}$, la mayor digestibilidad fue para T1 $(46.1 \%)$ y T2 $(44.5 \%)$ $(\mathrm{p}=0.0001)$. La mayor digestibilidad de la FDN fue para el T2 $(63.6 \%)(p=0.034)$. Sin embargo, la digestibilidad de la FDA fue mayor para T1 (52.4\%), T2 (55\%) y T4 (49.9\%) $(\mathrm{p}=0.0205)$, siendo superior al T3 con alrededor de 10\% (Cuadro 3).

\section{Discusión}

El mayor consumo voluntario de nutrientes (g/ave/día $\left.{ }^{1}\right)$ en los tratamientos con Azolla (Cuadro 2) se debió posiblemente, por un lado, a que la incorporación de Azolla en la dieta influyó en el aporte de fibra dietaria insoluble y, con ello, se podría haber aumentado el tránsito intestinal del alimento favoreciendo el consumo (Savón, 2002); y por otro lado, por la preferencia visual que tienen las aves por el alimento o tamaño de partícula del mismo para el caso de las dietas con Azolla. A diferencia de los mamíferos, las propiedades visuales y de textura del alimento tienen una influencia mayor en el consumo de alimento de las aves (Cooper, 1971). Estos resultados son consistentes a los reportados por Mahecha y Rosales (2005) y Andino y Patricia (2015), quienes mencionan que la inclusión de forraje en la dieta de las aves puede beneficiar el consumo de alimento.

Los mayores rendimientos de huevos (g/día) y mejor conversión alimenticia (Cuadro 2) pueden estar relacionados a una mayor digestión de los nutrientes, aspecto que fue observado en este estudio (Cuadro 3). El menor rendimiento productivo en T4 fue posiblemente debido a un menor aprovechamiento de los nutrientes (menor digestibilidad; Cuadro 4), ya que la Azolla contiene una mayor cantidad de fibra que acelera el tránsito intestinal. Estos resultados concuerdan con los reportados por Fuente et al. (2005), quienes mencionan que a mayor digestibilidad de los nutrientes se obtiene mayor rendimiento productivo.

Los resultados obtenidos en la digestibilidad aparente de la MS y MO (Cuadro 3) pudo deberse al contenido de carbohidratos presentes en las dietas (y que fue similar para todos los tratamientos: Cuadro 2). Sin embargo, la mayor digestibilidad de PC, FDN y FDA de las dietas con inclu- 
sión de $0 \%$ (T1) y 5\% (T2) de Azolla pudo deberse al menor aporte de fibra dietética en la dieta, en comparación a las dietas con niveles ascendentes de Azolla (T3 y T4), ya que esto provoca mayor tránsito del alimento por el tracto gastrointestinal, reduciendo la exposición del alimento a los procesos enzimáticos $\mathrm{y}$, con ello, una reducción de la digestibilidad (McDonald et al., 2011), aspecto parcialmente observado en este estudio (Cuadro 3). Aguilar et al. (2000), asimismo, mencionan que la inclusión de forrajes como parte de la dieta alimenticia en las aves disminuye la digestibilidad de nutrientes, disminuyendo a su vez, la conversión alimenticia y el rendimiento productivo.

\section{Conclusión}

La incorporación de Azolla en un 5\% en la dieta de codornices produce un efecto similar en la producción de huevos y conversión alimenticia que la dieta control, de modo que podría utilizarse como un producto no convencional de bajo costo.

\section{Agradecimiento}

Los autores agradecen a la Universidad Técnica de Ambato por el financiamiento de esta investigación, a través de la Dirección de Investigación y Desarrollo (DIDE), resolución CU-0135-P-2014

\section{Literatura Cittada}

1. Aguilar-Ramírez, J, Santos-Ricalde R, Pech-Martínez, V, Montes-Pérez $R$.

2000. Utilización de la hoja de chaya (Cnidoscolus chayamansa) y de huaxín (Leucaena leucocephala) en la alimentación de aves criollas. Rev Biomed 11: 17-24.

2. Andino P, Patricia S. 2015. Dietas con diferentes niveles de proteína más aminoácidos sintéticos en el comportamiento productivo de codornices de postura. Tesis de pregrado. Ecuador: Escuela Superior Politécnica del Chimborazo. $111 \mathrm{p}$.

3. [AOAC] Association of Official Analytical Chemists. 1990. Official methods of analysis. $15^{\text {th }}$ ed. Arlington, VA, USA: AOAC. $1230 \mathrm{p}$.

4. Bagh J, Panigrahi B, Panda N, Pradhan CR, Mallik BK, Majhi B, Rout SS. 2016. Body weight, egg production, and egg quality traits of gray, brown, and white varieties of Japanese quail (Coturnix coturnix japonica) in coastal climatic condition of Odisha. Vet World 9: 832-836. doi: 10.14202/ vetworld.2016.832-836

5. Çabuk M, Eratak S, Basmacioðlu Malayodlu H. 2014. Effects of dietary inclusion of lentil byproduct on performance and oxidative stability of eggs in laying quail. Scientific World J 2014: ID 742987. doi: $10.1155 / 2014 / 742987$

6. Cooper JB. 1971. Colored feed for turkey poults. Poult Sci 50: 1892-1893.

7. Dahouda M, Adjolohoun S, Montchowui EH, Senou M, Hounsou NMD, Amoussa S, et al. 2013. Growth performance of quails (Coturnix coturnix) fed on diets containing either animal or vegetable protein sources. Int J Poult Sci 12: 396-400. doi: 10.3923/ ijps.2013.396.400

8. Fuente B, Díaz A, Lecumberri J, Avila E. 2005. Necesidades de lisina y aminoácidos azufrados digestibles en ga1linas Leghorn Blancas. Vet Mex 36: 135145.

9. Iqbal MA, Roohi N, Akram M, Khan O. 2015. Egg quality and egg geometry influenced by mannan-oligosaccharides (MOS), a prebiotic supplementation in four close bred flocks of Japanese quail breeders (Coturnix coturnix japonica). Pakistan J Zool 47: 641-648.

10. Kocevski D, Nikolova N, Kuzelov A. 2011. The influence of strain and age on some egg quality parameters of commercial laying hens. Biotechnol Anim Husb 27: 1649-1658. 
11. Mahecha L, Rosales M. 2005. Valor nutricional del follaje de botón de oro (Tithonia diversifolia [Hemsl.] Gray), en la producción animal en el trópico. Livestock Res Rural Dev 17(9). [Internet]. Disponible en: http:// www.lrrd.org/lrrd17/9/mahe17100.htm

12. McDonald $P$, Edwards $R A$, Greenhalgh JFD, Morgan CA, Sinclair LA, Wilkinson RG. 2011. Animal nutrition. $7^{\text {th }}$ ed. Essex, UK: Pearson. $692 \mathrm{p}$.

13. Monira KN, Salahuddin M, Miah G. 2003. Effect of breed and holding period on egg quality characteristics of chicken. Int J Poult Sci 2: 261-263. doi: 10.3923/ ijps.2003.261.263

14. Özsoy AN, Aktan S. 2011. Estimation of genetic parameters for body weight and egg weight traits in Japanese quails. Trends Anim Vet Sci J 2: 17-20.
15. Panda B, Singh RP. 1990. Development in processing quail meat and eggs. Worlds Poult Sci J 46: 219234. doi: 10.1079/WPS19900022

16. Savón L. 2002. Alimentos altos en fibra para especies monogástricas. Caracterización de la matriz fibrosa y sus efectos en la fisiología digestiva. Rev Cuban Cienc Agr 36: 91-102.

17. Savory CJ, Gentle MJ. 1976. Changes in food intake and gut size in Japanese quail in response to manipulation of dietary fibre content. Br Poult Sci 17: 571-580. doi: 10.1080/00071667608416315

18. Vieira Filho JA, Garcia EA, Molino ADB, Santos TAD, Paz ICDLA, Baldo GADA. 2016. Productivity of Japanese quails in relation to body weight at the end of the rearing phase. Acta Sci Anim Sci 38: 213-217. doi: 10.4025/ actascianimsci.v38i2.29858 